\title{
Compression Deformation Behavior and Processing Map of Pure Copper
}

\author{
S. H. Huang, ${ }^{1}$ S. X. Chai, X. S. Xia, Q. Chen, and D. Y. Shu \\ Southwest Research Institute of Technology Engineering, Chongqing, China \\ 1 hsh82@163.com
}

To reveal compression deformation behavior of pure copper, the deformation characteristics of pure copper have been investigated by means of compression tests in the temperature range of $400-900^{\circ} \mathrm{C}$ and strain rate range of $0.001-1 \mathrm{~s}^{-1}$. The results show that the flow stress of pure copper increases with increasing strain rate and decreasing deformation temperature, which is characterized by work-hardening, dynamic recovery, dynamic recrystallization, and secondary work-hardening, etc. The activation energy of hot deformation is associated with deformation temperature and strain rate, and the average activation energy is calculated to be $303.8 \mathrm{~kJ} / \mathrm{mol}$. The flow stress prediction model based on $G A+B P$ possess, is in very good agreement with the true stress curve, which is of significance to the guidance of hot working of pure copper. The flow instability occurs in the intermediate strain rate region (0.01-0.1 $\mathrm{s}^{-1}$ ) base on the analysis of processing map, the high power dissipation correspond to the dynamic recrystallization. Appropriate reducing the deformation temperature or increasing the strain rate is beneficial for the grain refinement in the steady-state region of the processing map.

Keywords: pure copper, compression deformation, flow stress prediction, GA+BP possess, processing map.

Introduction. Excellent ductility and corrosion resistance of pure copper allows it to find a wide application value in the plastic forming field of high-end equipment product [1-5]. Flow stress of plastic deformation of pure copper is a comprehensive reflection, of which evolution in the interior microstructure of the deformable body, affected by the deformation temperature, deformation extent, strain rate and chemical composition of the pure copper. Currently, there are relatively considerable studies and reports on description of pure copper flow stress, such as hyperbolic sine function, Arrhenius-type constitutive model, and ANN model, but little on behavior of pure copper flow stress [6-14]. To accurately reveal the change rule of flow stress of pure copper in a plastic forming process, it is required to consider the impact of the strain on the flow stress.

In this paper, Gleeble-1500 thermal simulator was used to perform compression simulation of pure copper to investigate the change rule of flow stress during compressive deformation, to analyze the relationship between flow stress and strain, strain rate and deformation temperature, and to establish the flow stress predictive model of pure copper. Following the compression test, processing map is drawn to establish the safe and unsafe domain. This method can offer relatively more accurate flow stress data for numerical simulation while providing a reference for the development of the processing technique of pure copper.

1. Materials and Methods. The material used in this study was a pure copper bar, which was annealed at $420^{\circ} \mathrm{C}$ for $2 \mathrm{~h}$. The specimens with a diameter of $\varnothing 8 \times 12 \mathrm{~mm}$ were cut from the annealed bar, and the average initial grain size was about $180 \mu \mathrm{m}$. Compression test was carried out on a Gleeble-1500 thermal simulator in the temperature range of $400-900^{\circ} \mathrm{C}$ and strain rate range of $0.001-1 \mathrm{~s}^{-1}$, the total compression deformation was $60 \%$. The whole compression process was controlled by computer, with relevant data being collected in an automatic way.

2. Compression Test Flow Stress Behavior of Pure Copper. Figure 1 shows the stress-strain curve of pure copper under different conditions. When the deformation 


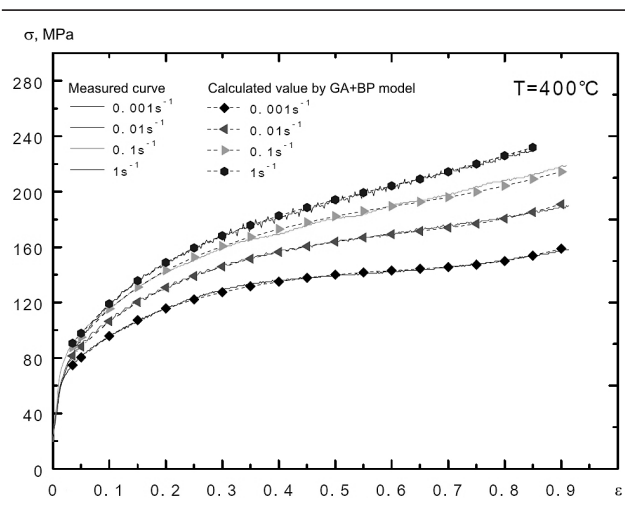

a

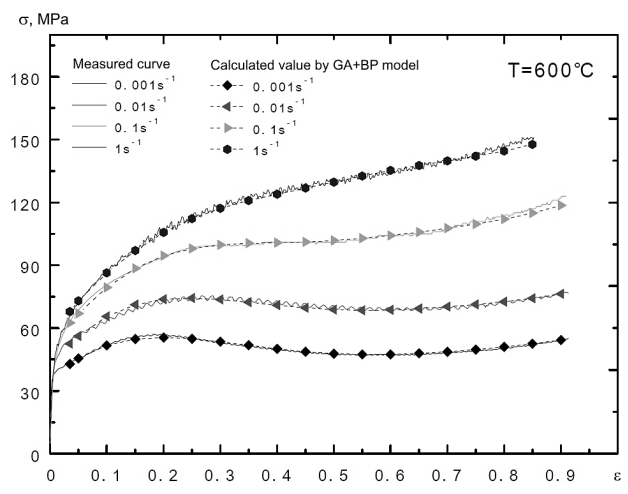

$\mathrm{c}$

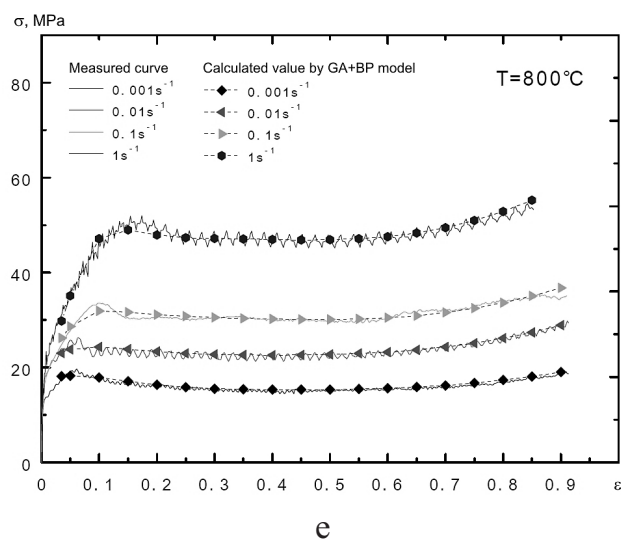

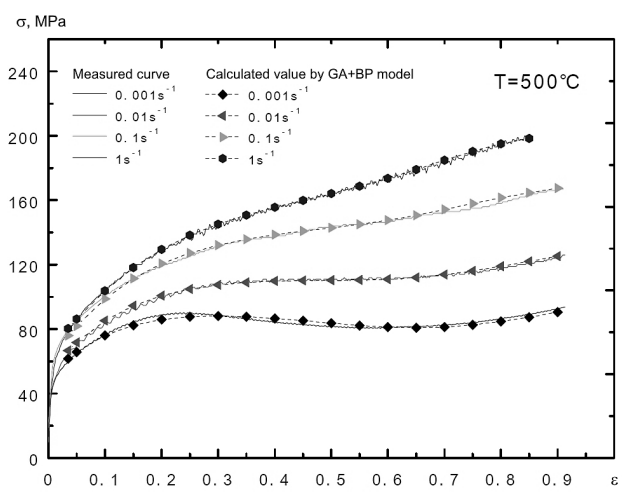

b

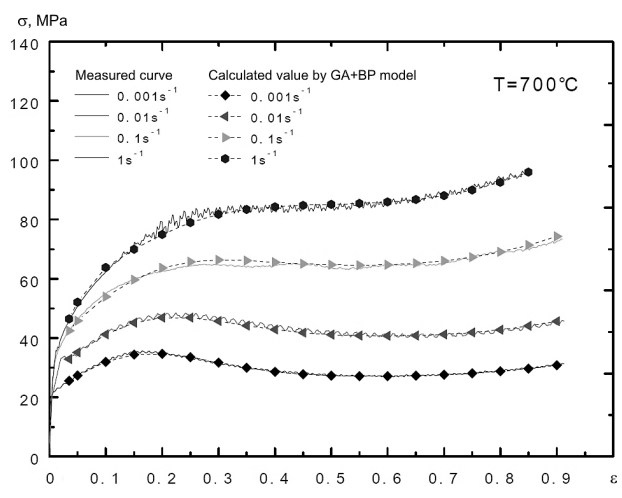

d

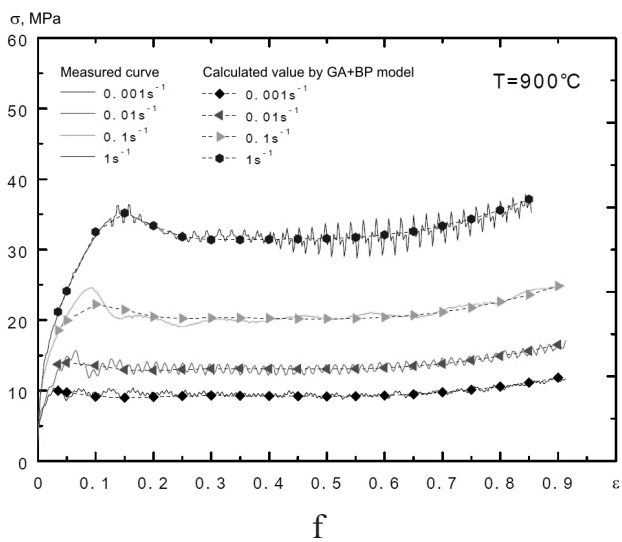

Fig. 1. Stress-strain curve of pure copper under different strain rates at temperatures of $400^{\circ} \mathrm{C}(\mathrm{a})$; $500^{\circ} \mathrm{C}(\mathrm{b}) ; 600^{\circ} \mathrm{C}(\mathrm{c}) ; 700^{\circ} \mathrm{C}(\mathrm{d}) ; 800^{\circ} \mathrm{C}(\mathrm{e}) ; 900^{\circ} \mathrm{C}$ (f).

temperature was comparatively low, work hardening dominated the deformation. With increasing of the strain rate, probability of deformation structure nucleation and growth decreased such that the dislocation density was enlarged, and the interaction between dislocations enhanced, which hindered further movement of the dislocations, showing that the deformation resistance was aggrandized with the increased strain. At elevated temperature and decreased deformation rate, thermal activation of the material was strengthened, average kinetic energy of the atoms increased, feature of dynamic recovery of the material became obvious gradually, and work hardening rate of the material decreased, till the 
softening and hardening effects were equal, flow stress curve reached the peak. Along with the temperature increased, the effect of dynamic recrystallization softening exceeded that of the work hardening, critical resolved shear stress of slip crystal decreased, enabling the flow stress to be lowered with increased strain. Meanwhile after the deformation exceeded 0.7 , the material exhibited the feature of secondary work hardening and increased dislocation density, and the hardening rate was greater than the softening rate. It can be seen from the curve shape that the material is characterized by work hardening, dynamic recovery and dynamic recrystallization, etc. proven to be a sensitive material associated with temperature and strain rate.

One of the features of high-temperature plastic deformation is that the strain rate is controlled by the thermal activation process. The impact of the deformation temperature and strain rate on the flow stress of metal material can be expressed quantitatively by the following creep equations [7-10]:

$$
\begin{gathered}
A_{1} \sigma^{n}=\dot{\varepsilon} \exp \left(\frac{Q}{R T}\right)=Z, \\
A_{2} \exp (\beta \sigma)=\dot{\varepsilon} \exp \left(\frac{Q}{R T}\right)=Z, \\
A[\sinh (\alpha \sigma)]^{n^{\prime}}=\dot{\varepsilon} \exp \left(\frac{Q}{R T}\right)=Z,
\end{gathered}
$$

where $Z$ is Zener-Hollomon parameter, whose physical meaning is that temperature compensated strain rate factors, $\mathrm{s}^{-1}, A_{1}, A_{2}, A, n, \beta, \alpha$, and $n^{\prime}$ are constants independent of temperature, $\beta=a n, \sigma$ is the high temperature flow peak stress, $\dot{\varepsilon}$ is the strain rate, $T$ is the deformation temperature, $\mathrm{K}, R$ is molar gas constant, $8.314 \mathrm{~J} /(\mathrm{mol} \cdot \mathrm{K})$, $Q$ is the hot deformation activation energy, which reflects difficulty level of hot deformation of the material, $\mathrm{kJ} / \mathrm{mol}$. Investigation shows that Eqs. (1) and (2) apply to low and high stress deformation respectively, and Eq. (3) is the general form of Eqs. (1) and (2). In the case of $\alpha \sigma<0.8$, Eq. (3) can be simplified as Eq. (1); at $\alpha \sigma>1.2$, Eq. (3) can be simplified as Eq. (2). From Eqs. (1)-(3), logarithm is taken respectively, then

$$
\begin{gathered}
\ln Z=\ln \dot{\varepsilon}+\frac{Q}{R T}=\ln A_{1}+n \ln \sigma, \\
\ln Z=\ln \dot{\varepsilon}+\frac{Q}{R T}=\ln A_{2}+\beta \sigma, \\
\ln Z=\ln \dot{\varepsilon}+\frac{Q}{R T}=\ln A+n^{\prime} \ln [\sinh (\alpha \sigma)] .
\end{gathered}
$$

When deformation temperature $T$ or strain rate $\dot{\varepsilon}$ is a constant value, peak stress $\sigma_{p}$ presents a nearly linear relationship with $\log$ value of strain rate $\dot{\varepsilon}$ or reciprocal value of temperature, as shown in Fig. 2, indicating that pure copper accords with the Arrhenius equation. For $\beta$ value, average value of the last 3 straight slopes in Fig. $2 \mathrm{a}$ is taken, then $\beta=0.1154$, the $R$ for data of $\sigma_{p}-\ln \dot{\varepsilon}$ are $0.98207,0.98274,0.97438,0.98818,0.99343$, 0.99609 , and 1 , which suggest that the relationship between $\sigma_{p}$ and $\ln \dot{\varepsilon}$ shows a good fitting degree. For $n$ value, mean value of the first 4 straight slopes in Fig. $2 \mathrm{~b}$ is taken, then $n=6.7847$ and $\alpha=\beta / n=0.017 \mathrm{MPa}^{-1}$. 


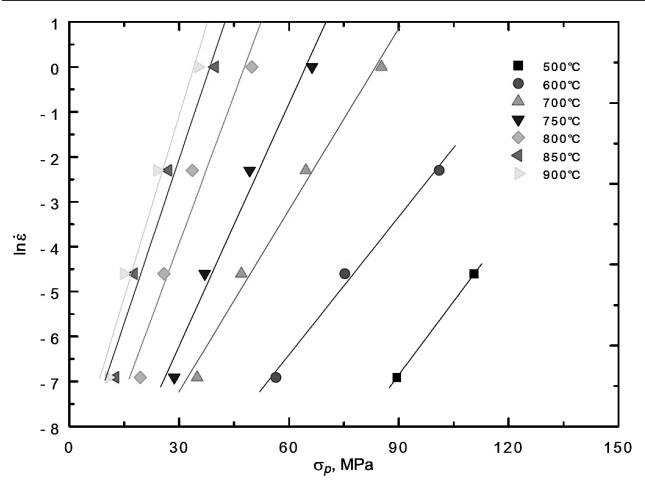

a

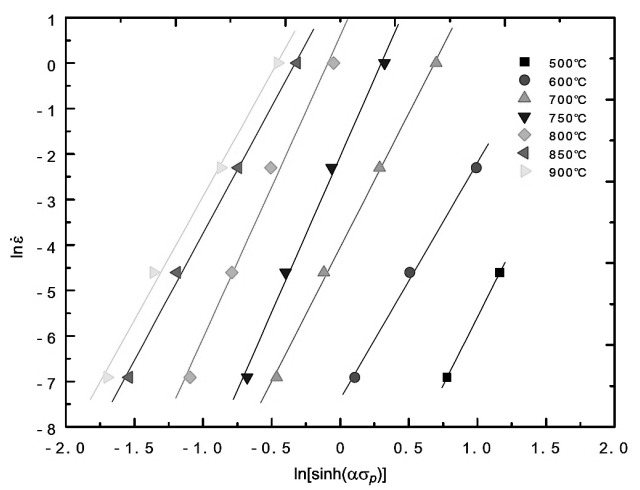

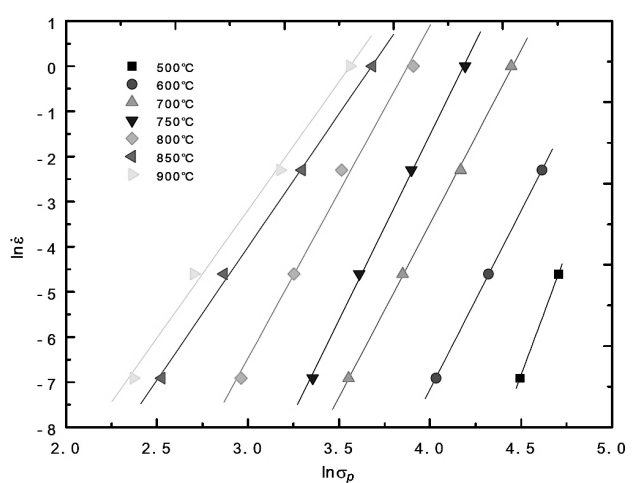

$\mathrm{b}$

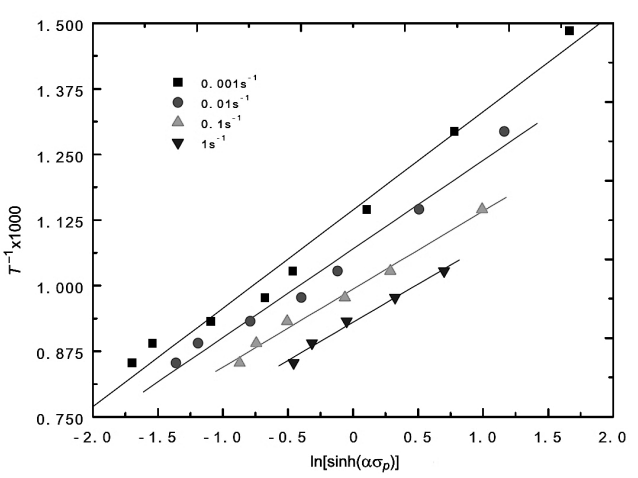

d

Fig. 2. Relationship between $\sigma_{p}$ and $\ln \dot{\varepsilon}$ (a); $\ln \sigma_{p}$ and $\ln \dot{\varepsilon}(\mathrm{b}) ; \ln \left[\sinh \left(\alpha \sigma_{p}\right)\right]$ and $\ln \dot{\varepsilon}$ (c); $\ln \left[\sinh \left(\alpha \sigma_{p}\right)\right]$ and $T^{-1}(\mathrm{~d})$.

It is known from Eq. (6) that the relationship between $\partial \ln \dot{\varepsilon}$ and $\partial(1 / T)$ is shown in Eq. (7). When the temperature $T$ is constant, for $\left[\frac{\partial \ln \dot{\varepsilon}}{\partial(\ln [\sinh (\alpha \sigma)])}\right]_{T}$ average slope obtained from 7 curves in Fig. 2c is around 5.93904; when the strain rate $\dot{\varepsilon}$ is constant, for $\left[\frac{\partial(1 / T)}{\partial(\ln [\sinh (\alpha \sigma)])}\right]_{\dot{\varepsilon}}$ average slope obtained from 4 curves in Fig. $2 \mathrm{~d}$ is approximately 0.000163 . Then, obtained by Eq. (8) the macroscopic activation energy $Q=303.8 \mathrm{~kJ} / \mathrm{mol}$,

$$
\begin{gathered}
\frac{\partial \ln \dot{\varepsilon}}{\partial(1 / T)}=\frac{Q}{R}=\left[\frac{\partial \ln \dot{\varepsilon}}{\partial(\ln [\sinh (\alpha \sigma)])}\right]_{T} /\left[\frac{\partial(1 / T)}{\partial(\ln [\sinh (\alpha \sigma)])}\right]_{\dot{\varepsilon}} \\
Q=R\left[\frac{\partial \ln \dot{\varepsilon}}{\partial(\ln [\sinh (\alpha \sigma)])}\right]_{T} /\left[\frac{\partial(1 / T)}{\partial(\ln [\sinh (\alpha \sigma)])}\right]_{\dot{\varepsilon}} .
\end{gathered}
$$

3. Establishment of Flow Stress Prediction Model Based on GA+BP Possess. As there is relatively great difference between flow stress $\sigma$, deformation temperature $T$, strain rate $\dot{\varepsilon}$, and strain $\varepsilon$ in terms of data dimension, to avoid the fact that the numerical values are too centralized on some neurons and weights that lead to lower computational accuracy of the model during iteration calculation of network model, it is required to perform 
normalization processing of flow stress $\sigma$, deformation temperature $T$, and strain rate $\dot{\varepsilon}$, as shown in Eq. (9):

$$
\begin{gathered}
T^{\prime}=0.035+0.865\left(\frac{T-T_{\min }}{T_{\max }-T_{\min }}\right), \quad \sigma^{\prime}=\ln \sigma, \\
\dot{\varepsilon}^{\prime}=0.035+0.865\left(\frac{\ln \dot{\varepsilon}-\ln \dot{\varepsilon}_{\text {min }}}{\ln \dot{\varepsilon}_{\text {max }}-\ln \dot{\varepsilon}_{\text {min }}}\right) .
\end{gathered}
$$

By features of high-temperature compression deformation of pure copper, it was determined that the network inputs were the deformation temperature $T^{\prime}$, the strain rate $\dot{\varepsilon}^{\prime}$, and the strain $\varepsilon$, respectively, and the flow stress $\sigma^{\prime}$ was the network output. GA genetic algorithm was used to optimize weights and thresholds, BP neural network training and prediction $[13,14]$. The author of this paper has prepared relevant procedures, with the number of initial hidden layer neurons being 20 and maximum genetic algebra 30. Network training function is Trainbr, the weight learning function Learngdm and training objectives $1 \cdot 10^{-12}$. The optimized weights and thresholds are shown separately in Fig. 3a and $3 \mathrm{~b}$. Figure $3 \mathrm{c}$ shows the comparison between the actual stress of pure copper and predicted stress of the model. As can be found the predicted stress and the actual stress fitted very well. Minimum RMSE of logarithmic stress was 0.58936, indicating that the GA+BP network model proposed by the author could well predict compression deformation behavior of pure copper and stress-strain curve, as shown in Fig. 1.

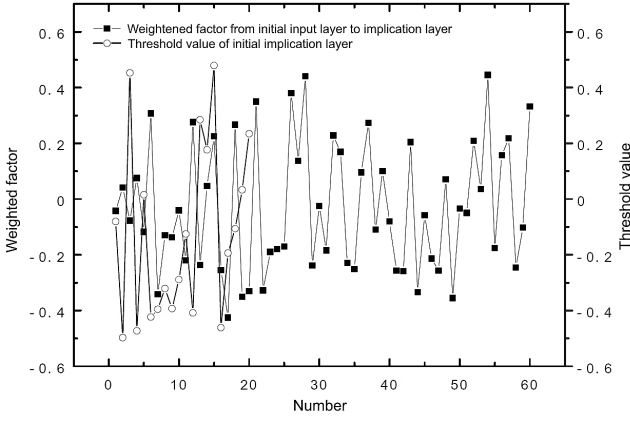

a

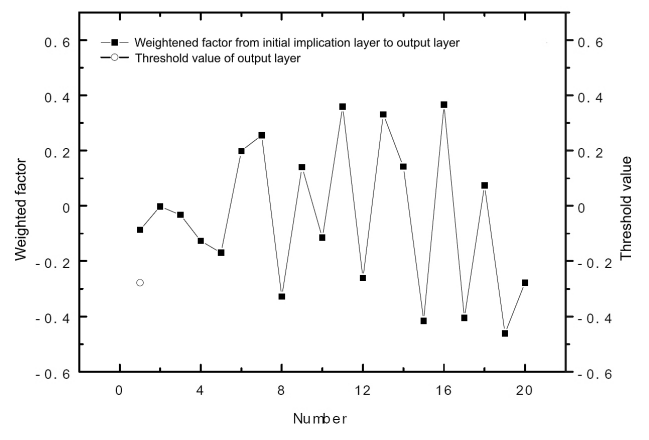

b

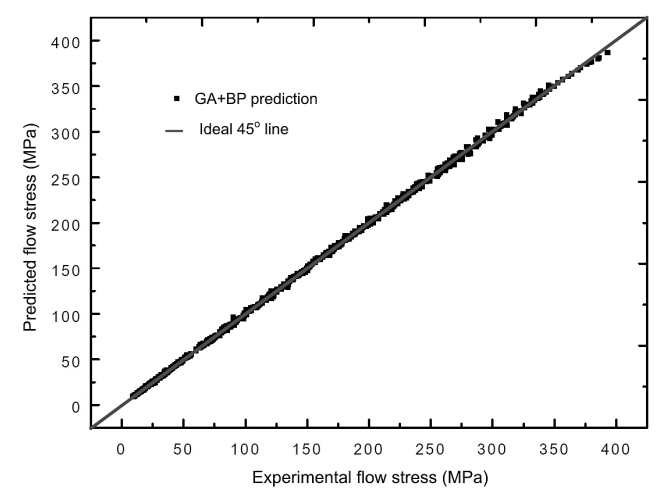

C

Fig. 3. Calculation results based on GA+BP network model: (a) optimal weights and thresholds between initial input layer and implication layer; (b) optimal weights and thresholds between initial implication layer and output layer; (c) comparison between actual and predicted stress. 
4. Discussion. Figure 4 shows the relationship between deformation activation energy and temperature at different strain rates. The change rule of pure copper deformation activation energy under different strain rates is identical to that of hardening exponent $n$, indicating that the dislocation mechanism and its change rule are identical during the deformation. Going up or going down of the activation energy curve indicates there are new dislocation mechanisms involved in the process of deformation. In the range of $500-600^{\circ} \mathrm{C}$, the lowered deformation activation energy indicates that softening deformation mechanism is in action. In the range of $600-750^{\circ} \mathrm{C}$, deformation activation energy increases sharply, which indicates that a variety of interactions of dislocation mechanism, hardening and softening, diffusion activation and effective stress constitute a complex movement of dislocations during deformation, where the work hardening is dominant. In the range of $750-900^{\circ} \mathrm{C}$, deformation activation energy declines sharply, exhibiting an obvious effect of softening mechanism of deformation and lowered energy barrier of dislocation activation. Meanwhile, the deformation activation energy increases with increasing of strain rate $\dot{\varepsilon}$. Accordingly, in the process of hot working, it is required to use appropriate strain rate with the premise of minimum energy consumption to minimize the impact of the work hardening.

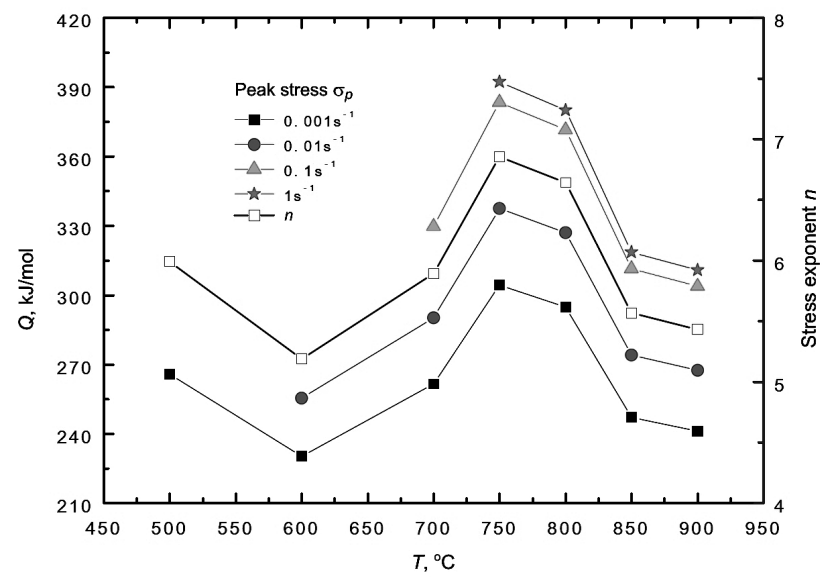

Fig. 4. Relationship between deformation activation energy and temperature under different strain rates.

Under condition of given strain $\varepsilon$ and deformation temperature $T$, strain rate sensitivity exponent $m$ can be defined by the rate of change of dissipation amount function $G$ and dissipation co-function $J$ [15-18], as shown in Eq. (10):

$$
m=\frac{d J}{d G}=\left[\frac{\partial \ln (\sigma)}{\partial \ln (\dot{\varepsilon})}\right]_{\varepsilon, T} .
$$

The dimensionless power dissipation efficiency factor $\eta$ can be described by the strain rate sensitivity exponent $m$, as shown in Eq. (11). To ensure the accuracy of $m$, a cubic equation function is used to fit the relationship between flow stress $\ln \sigma$ and $\ln \dot{\varepsilon}$. Power dissipation efficiency factor $\eta$ constitutes the power dissipation map with the variation of temperature and strain rate,

$$
\eta=\frac{J}{J_{\max }}=\frac{2 m}{m+1} .
$$


According to the principle of irreversible thermodynamics, the dimensionless parameter $\xi(\dot{\varepsilon})$ can be used to express continuous instability criterion at large plastic deformation, as shown in Eq. (12). With the deformation temperature $T$ and strain rate $\dot{\varepsilon}$ as variables, non-steady flow region with parameter $\xi(\dot{\varepsilon})$ smaller than zero constitutes the instability map,

$$
\xi(\dot{\varepsilon})=\frac{\partial \ln \left(\frac{m}{m+1}\right)}{\partial \ln \dot{\varepsilon}}+m<0 .
$$

The power dissipation map and the instability map are superimposed together to form DMM processing map corresponding to different true strain capacity $\varepsilon$, where the shadow area is generally expressed as instability region, and the digital on equivalent line represents the power dissipation factor.

Figure 5 is the processing maps of the pure copper at strains of 0.2 and 0.6 . As can be known with decreased deformation temperature and increased strain, the flow instability region becomes obvious larger, possibly due to the condition of severe stress concentration that leads to shear cracking [15-18]. At strain of 0.2 , the material exhibits a good plasticity at low strain rate $\left(<0.01 \mathrm{~s}^{-1}\right)$. At high strain rate $\left(\geq 0.1 \mathrm{~s}^{-1}\right)$ the range of hot working is relatively narrow, while at the intermediate strain rate $\left(0.01-0.1 \mathrm{~s}^{-1}\right)$ flow instability most likely takes place, probably because of the relatively high deformation temperature at $400-700^{\circ} \mathrm{C}$. Under moderate strain rate, grain boundary of the material is even more prone to slip, and then a severe stress concentration is generated at the interface, thus leading to cracking of the interface. Whereas the stress concentration generated due to interface slip under low strain rate has enough time to be released by the way such as diffusion; under high strain rate, deformation rate of the matrix is faster than the rate of interface slip, here the effect of slip can be ignored, which results in no cracking.

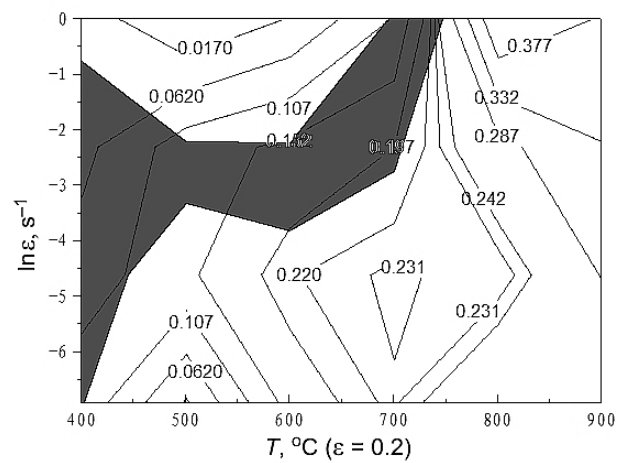

a

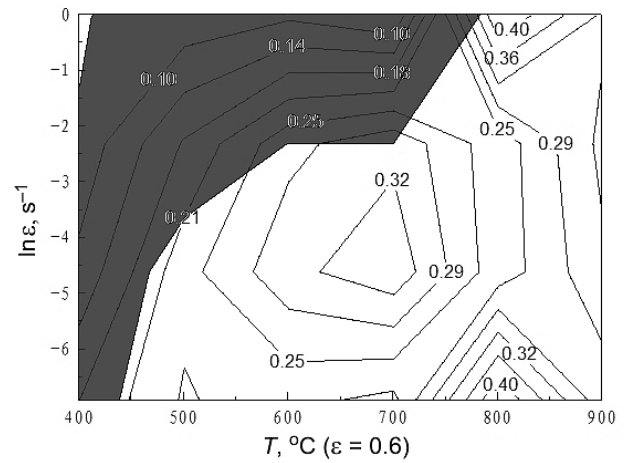

$\mathrm{b}$

Fig. 5. Processing maps of the pure copper at strains of 0.2 (a) and 0.6 (b).

When the deformation degree increases to 0.6 , the value of the power dissipation efficiency factor $\eta$ becomes greater. The large power dissipation efficiency represents special microstructure or flow instability mechanism, that is to say, the dynamic recovery or dynamic recrystallization may probably occur, and local flow instability may also take place. Results of microstructure in Fig. 6a indicate in the processing map the deformation mechanism of the region with a relatively high power dissipation factor is dynamic recrystallization. It is possible to obtain finer grain structure by properly decreasing the deformation temperature or increasing the strain rate at the steady-state region of the processing map, as shown in Fig. 6b. 


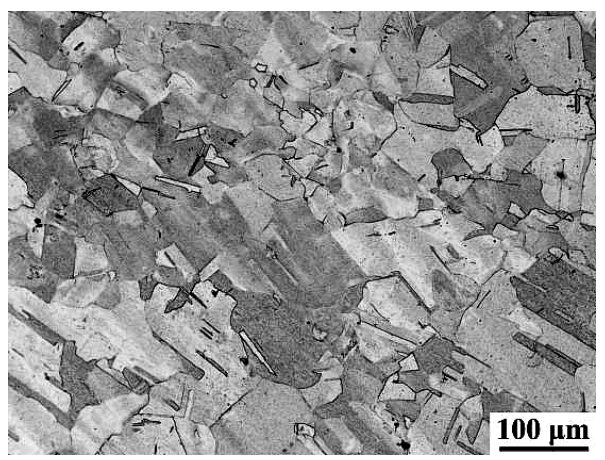

a

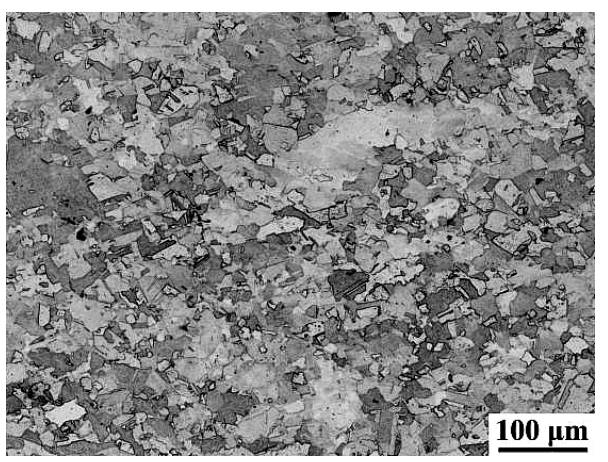

b

Fig. 6. Microstructure under condition of $T=800^{\circ} \mathrm{C}, \dot{\varepsilon}=0.001 \mathrm{~s}^{-1}$ (a); $T=500^{\circ} \mathrm{C}, \dot{\varepsilon}=1 \mathrm{~s}^{-1}$ (b).

Conclusions. High temperature compression deformation true stress-true strain curve of pure copper is characterized by work-hardening, dynamic recovery, dynamic recrystallization and secondary work hardening, etc. In case the deformation temperature is constant, the flow stress increases with increasing of the strain rate. When strain rate is constant, the flow stress decreases with increasing of the deformation temperature. It belongs to temperature and rate-sensitive material.

Hot deformation peak stress of pure copper alloy meets the hyperbolic sine constitutive relation, and the hot deformation activation energy is associated with deformation temperature and strain rate, whose average hot deformation activation energy is $303.8 \mathrm{~kJ} / \mathrm{mol}$.

Analysis of DMM processing map shows that the range of flow instability region becomes large notably with decrease deformation temperature and increase strain. The flow instability occurs in the intermediate strain rate region $\left(0.01-0.1 \mathrm{~s}^{-1}\right)$ base on the analysis of processing map, the high power dissipation correspond to the dynamic recrystallization. Appropriate reducing the deformation temperature or increasing strain rate is beneficial for the grain refinement in the steady-state region of the processing map.

Acknowledgments. This research is funded by Chongqing Research Program of Basic Research and Frontier Technology (No. CSTC2015jcyjB0471).

1. W. H. Tian, A. L. Fan, H. Y. Gao, et al., "Comparison of microstructures in electroformed copper liners of shaped charges before and after plastic deformation at different strain rates," Mater. Sci. Eng. A, 350, 160-167 (2003).

2. A. L. Fan, S. K. Li, W. H. Tian, and F. C. Wang, "Comparison of microstructures in electroformed and spin-formed copper liners of shaped charge undergone high-strainrate deformation," Trans. Nonferrous Met. Soc. China, 17, 1447-1450 (2007).

3. A. L. Fan, S. K. Li, and W. H. Tian, "Grain growth and texture evolution in electroformed copper liners of shaped charges," Mater. Sci. Eng. A, 474, 208-213 (2008).

4. O. Ozhoga-Maslovskaja, K. Naumenko, H. Altenbach, and O. Prygorniev, "Micromechanical simulation of grain boundary cavitation in copper considering nonproportional loading," Comput. Mater. Sci., 96, 178-184 (2015).

5. Z. L. Wang, H. P. Wang, Z. H. Hou, et al., "Dynamic consolidation of W-Cu nano-alloy and its performance as liner materials," Rare Metal Mat. Eng., 43, No. 5, 1051-1055 (2014). 
6. C. W. Tan, S. N. Xu, L. Wang, et al., "Deformation behavior of AZ31 magnesium alloy at different strain rates and temperatures," Trans. Nonferrous Met. Soc. China, 17, 347-352 (2007).

7. F. Zhang, J. Shen, X. D. Yan, and J. L. Sun, "Constitutive analysis to predict high-temperature flow stress in 2099 Al-Li alloy," Rare Metal Mat. Eng., 43, No. 6, 1312-1318 (2014).

8. W. Li, H. Li, Z. X. Wang, and Z. Q. Zheng, "Constitutive equations for high temperature flow stress prediction of Al-14Cu-7Ce alloy," Mater. Sci. Eng. A, 528, 4098-4103 (2011).

9. M. A. Jabbari Taleghani, E. M. Ruiz Navas, M. Salehi, and J. M. Torralba, "Hot deformation behaviour and flow stress prediction of 7075 aluminium alloy powder compacts during compression at elevated temperatures," Mater. Sci. Eng. A, 534, 624-631 (2012).

10. H. Mirzadeh and A. Najafizadeh, "Flow stress prediction at hot working conditions," Mater. Sci. Eng. A, 527, 1160-1164 (2010).

11. A. K. Maheshwari, "Prediction of flow stress for hot deformation processing," Comput. Mater. Sci., 69, 350-358 (2013).

12. S. Serajzadeh and A. K. Taheri, "Prediction of flow stress at hot working condition," Mech. Res. Commun., 30, 87-93 (2003).

13. G. Z. Quan, W. Q. Lv, Y. P. Mao, et al., "Prediction of flow stress in a wide temperature range involving phase transformation for as-cast Ti-6Al-2Zr-1Mo-1V alloy by artificial neural network," Mater. Des., 50, 51-61 (2013).

14. A. K. Gupta, S. K. Singh, S. Reddy, and G. Hariharan, "Prediction of flow stress in dynamic strain aging regime of austenitic stainless steel 316 using artificial neural network," Mater. Des., 35, 589-595 (2012).

15. X. S. Xia, Q. Chen, K. Zhang, et al., "Hot deformation behavior and processing map of coarse-grained Mg-Gd-Y-Nd-Zr alloy," Mater. Sci. Eng. A, 587, 283-290 (2013).

16. F. A. Slooff, J. S. Dzwonczyk, J. Zhou, et al., "Hot workability analysis of extruded AZ magnesium alloys with processing maps," Mater. Sci. Eng. A, 527, 735-744 (2010).

17. G. Meng, B. L. Li, H. M. Li, et al., "Hot deformation and processing maps of an Al-5.7 wt.\% Mg alloy with erbium," Mater. Sci. Eng. A, 517, 132-137 (2009).

18. Y. V. R. K. Prasad and K. P. Rao, "Effect of homogenization on the hot deformation behavior of cast AZ31 magnesium alloy," Mater. Des., 30, 3723-3730 (2009). 